\title{
Panorama epidemiológico de dengue en México 2000-2019
}

\section{Epidemiological panorama of dengue in Mexico 2000-2019}

\author{
José Luis Arredondo-García, Carmen Gabriela Aguilar-López Escalera, Juan José Aguilar Lugo-Gerez, \\ Neydi Osnaya-Romero, Gabriela Pérez-Guillé, Humberto Medina-Cortina \\ Unidad de Investigación Clínica. Instituto Nacional de Pediatría (INP), Ciudad de México, México.
}

RESUMEN

\begin{abstract}
El dengue es una enfermedad infecciosa viral que sigue siendo un problema de salud pública en México y el mundo. Este trabajo describe los casos confirmados (incidencia nacional) en México entre 2010 y 2019, tomando como referencia lo reportado por el SINAVE. La información recolectada reflejó cinco principales brotes en 2007, 2009, 2012, 2013 y el más reciente en 2019 con 41,505 casos confirmados al cierre del año, $226 \%$ más en comparación con el año 2018 (12,706). Este incremento de casos también se observó en otros países de la Región de las Américas, alcanzando hasta la semana epidemiológica 42 el mayor número de casos registrados en la historia $(2,733,635)$. Estos datos demuestran que, a pesar de las estrategias de control, el virus presenta un patrón cíclico de brotes que puede coincidir en otras regiones, por lo que resulta imperante reforzar las estrategias de vigilancia, diagnóstico y control que mitiguen su impacto.
\end{abstract}

Palabras clave: Dengue, dengue grave, dengue con signos de alarma, dengue no grave incidencia, México.

\section{INTRODUCCIÓN}

El dengue es una enfermedad infecciosa viral que sigue siendo un problema de salud pública a nivel mundial debido al impacto en la morbilidad, mortalidad y repercusión económica. ${ }^{1,2}$ Alrededor de 3 mil millones de personas habitan en áreas endémicas y se calcula cerca de 400 millones de casos nuevos cada año, aunque sólo $25 \%$ cursan sintomáticos. ${ }^{3}$

En México, el dengue es la infección viral transmitida, con mayor frecuencia, por artrópodos ${ }^{4}$ y al igual

Financiamiento: Ninguno.

Conflicto de intereses: Ninguno.

https://dx.doi.org/10.35366/94418

Rev Latin Infect Pediatr 2020; 33 (2): 78-83

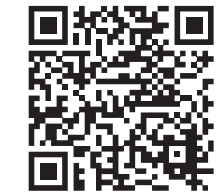

ABSTRACT

Dengue is a viral infectious disease that remains a public health problem in Mexico and the world. This report describes confirmed cases in Mexico (national incidence), between 2000 and 2019, based on reports published by the SINAVE. The data collected show five main outbreaks in 2007, 2009, 2012, 2013 and the most recent in 2019 with 41,505 confirmed cases at the end of the year, 226\% more than in 2018 (12,706). This increase was also observed in other countries of the Americas, reaching since week 42 , the highest number of cases recorded in history $(2,733,635)$. These data demonstrate that, despite control strategies, the virus has a cyclical pattern of outbreaks that may coincide in other regions, being necessary to have better surveillance, diagnosis and control strategies that mitigate the impact.

Keywords: Dengue, severe dengue, dengue with alarm signs, dengue not severe incidence, Mexico.

que otros países de América Latina, como Brasil, Honduras y Colombia, contribuye en gran proporción a los casos de dengue reportados en la región. ${ }^{5}$ En las últimas décadas, a pesar de las estrategias de control, se han documentado distintos brotes en México, por lo cual la infección por virus del dengue se ha catalogado como uno de los problemas actuales más importantes de salud pública en el país. ${ }^{6}$

El virus del dengue pertenece al género Flavivirus, de la familia Flaviviridae del grupo de los Arbovirus y está conformado por cuatro serotipos: DENV-1, DENV-2, DENV-3 y DENV-4. ${ }^{7}$ La infección por un serotipo produce inmunidad permanente contra la reinfección por ese serotipo. Sin embargo, una infección sucesiva con dos serotipos diferentes es un factor de riesgo para desarrollar las formas graves de la enfermedad. ${ }^{8}$ Sus vectores son los mosquitos 
Rev Latin Infect Pediatr 2020; 33 (2): 78-83

Aedes aegypti y $A$. albopictus, los cuales crecen en cuerpos de agua en las regiones tropicales y subtropicales de todo el mundo. Las principales complicaciones que surgen durante el cuadro de dengue son derivadas de alteraciones de la hemostasia, fuga de plasma hacia el espacio intersticial y daño orgánico, principalmente hepático y del sistema nervioso central. ${ }^{9}$ La letalidad del dengue cuando se presentan complicaciones es de $2-5 \%$ si se recibe tratamiento, pero escala hasta el $20 \%$ cuando se deja sin tratar; mientras que la letalidad de los casos no complicados es menor a $1 \%{ }^{3}$

La OMS desarrolló en 2009 una nueva clasificación del dengue. ${ }^{10}$ Esta clasificación fue incorporada a los reportes epidemiológicos del Sistema Nacional de Vigilancia Epidemiológica (SINAVE) en México en 2016 y organiza los casos en dos categorías: dengue grave (DG) y dengue no grave (DNG); con una tercera subcategoría dentro del DNG denominada dengue con signos de alarma (DCSA).

En México, el Sistema Nacional de Vigilancia Epidemiológica creado desde 1990, es la plataforma que integra la información epidemiológica en el país, la cual proporciona información sobre el estado de salud de la población, riesgos, necesidades y el efecto de los planes y programas de intervención. El sistema de recopilación de datos lo componen alrededor de 20,005 unidades de atención de la salud que envían la información a la Dirección General de Epidemiología (DGE) para favorecer la recolección continua, oportuna y confiable de la información. La vigilancia del dengue es obligatoria e incluye casos sospechosos y confirmados, los cuales requieren del diagnóstico de laboratorio por serología o aislamiento del virus. La notificación de casos también es obligatoria y es reportada de forma semanal a través de los Boletines Epidemiológicos (BE) publicados en la página del SINAVE. ${ }^{1}$

Durante el año 2019 se observó un incremento en la incidencia del dengue a nivel global. Entre los factores involucrados está el fenómeno del calentamiento global, que amplía el hábitat del vector, el pobre tratamiento de cuerpos de agua que favorece su crecimiento y los incrementos en la densidad poblacional, los fenómenos migratorios y los movimientos humanos, que en conjunto aumentan las posibilidades de transmisión. ${ }^{12-14}$

Es necesario estudiar a fondo el panorama epidemiológico actual de dengue en México para intentar conocer los factores que están llevando al aumento observado en la incidencia y con ello lograr el desarrollo de estrategias preventivas eficaces.

El objetivo del presente trabajo fue describir el comportamiento epidemiológico de casos confirmados de dengue (incidencia nacional) en las últimas dos décadas en México (2010-2019) para identificar los principales brotes y comparar con el incremento de casos más reciente durante 2019 que se presentó a nivel regional y mundial.

\section{MATERIAL Y MÉTODOS}

Se realizó una recolección de los casos confirmados de dengue publicados en los BE del SINAVE para cada una de las 52 semanas epidemiológicas (SE) entre 2000 y $2019 .{ }^{15,16}$ De igual forma, se obtuvieron los casos por sexo y entidad federativa. Además, se calculó la tasa de incidencia (TI) anual de casos confirmados de dengue a nivel nacional. La recopilación de los datos se realizó a través de una tabla dinámica generada en una hoja de cálculo de Excel (Microsoft Corp., Redmond, WA).

Para los años 2000 a 2015 se extrajeron los datos confirmados de fiebre por dengue (FD) y fiebre hemorrágica por dengue (FHD). A partir de 2016 a la fecha, se comenzó a utilizar la clasificación propuesta por la OMS (publicada en 2009): dengue no grave (DNG), dengue con signos de alarma (DCSA) y dengue grave (DG).

\section{RESULTADOS}

Después de la reintroducción de dengue en México con los brotes reportados en 1980 y 1997, se tuvieron cinco años de baja endemia (2000-2004); sin embargo, en las últimas dos décadas se han presentado nuevos brotes correspondientes a los años 2007, 2009, 2012, 2013 y el más reciente en 2019 (Figuras 1 y 2), periodo en el cual (2000-2019) se han registrado más de 518,000 casos confirmados de dengue de acuerdo con lo publicado en los $B E$ y reportes semanales de dengue emitidos por el SINAVE.

Comenzando con el primer brote de este periodo, en 2007 se presentaron 48,456 casos confirmados ( $F D=40,559$ y $\mathrm{FHD}=7,897)$ y una $\mathrm{TI}$ de 44.6, con mayor número de casos en Veracruz, Oaxaca, Guerrero, Quintana Roo y Nuevo León. El sexo más afectado fue el femenino con $57 \%$ de los casos confirmados de FD y en los casos de FHD 51\% fue del sexo femenino y $49 \%$ del masculino. 


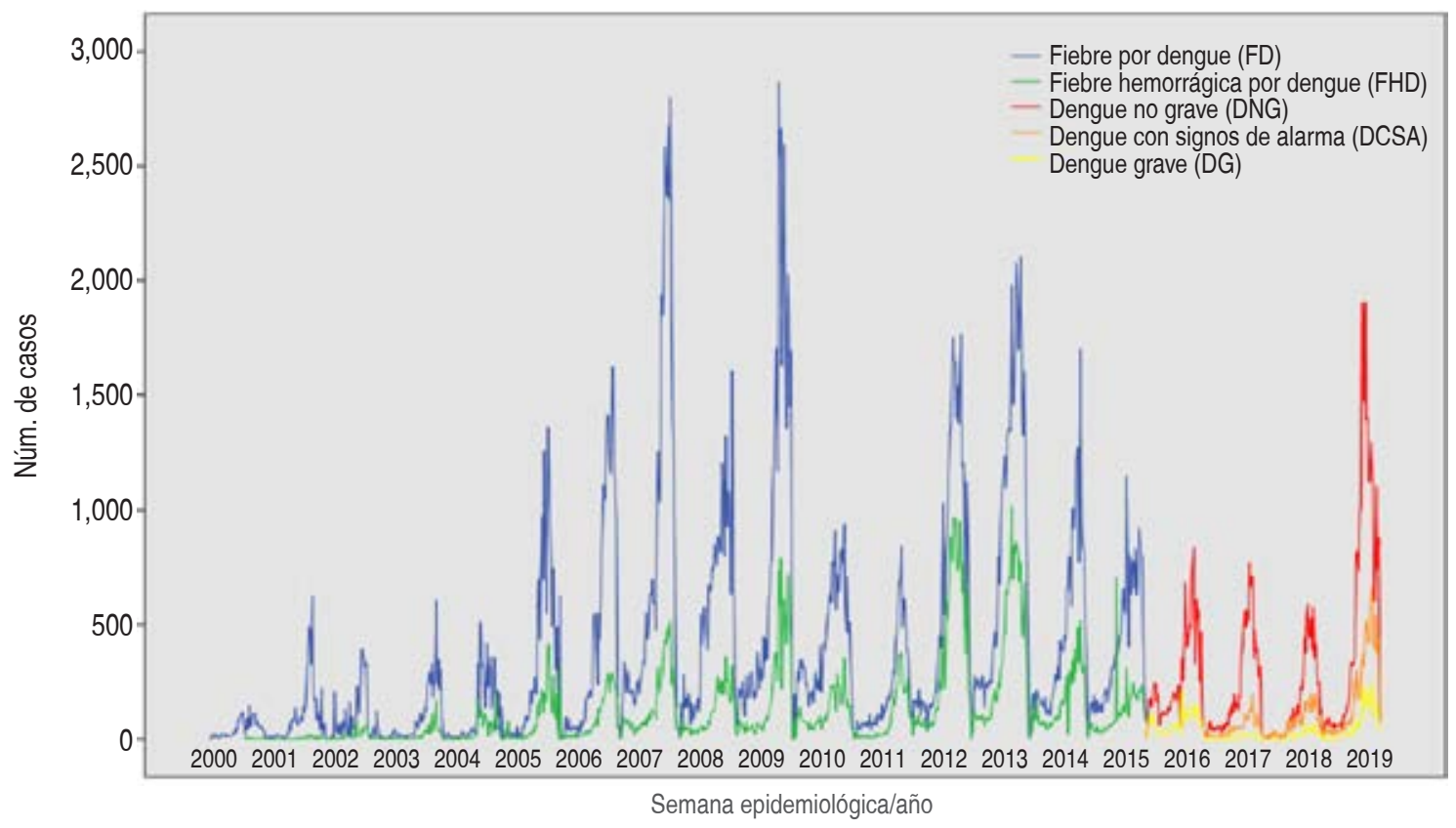

Figura 1: Casos confirmados de dengue en México de 2000 a 2019..$^{15-17}$

Nota: a partir de 2016 comienzan a reportarse los casos en los boletines epidemiológicos utilizando la clasificación de dengue no grave, dengue con signos de alarma, dengue grave.

En el segundo pico, en 2009 se reportó un aproximado de 52,534 casos confirmados (FD $=41,972$ y $\mathrm{FHD}=10,562$ ) y una TI de 46.8, siendo Veracruz, Guerrero, Nayarit, Jalisco y Colima los estados con más casos. El $54 \%$ de los casos de FD correspondieron al sexo femenino, mientras que en los casos de FHD $49 \%$ al sexo femenino y $51 \%$ al masculino.

Para el brote de 2012 se reportaron 50,368 casos de dengue confirmados (FD = 32,662 y FHD $=17,706)$ y una $\mathrm{TI}=43$, los estados de Veracruz, Yucatán, Morelos, Guerrero y Chiapas presentaron más casos. El $55 \%$ de los casos confirmados de FD correspondió a las mujeres, mientras que $50 \%$ de los casos de FHD en los hombres.

En 2013 fueron 62,330 los casos confirmados reportados $(\mathrm{FD}=43,663$ y $\mathrm{FHD}=18,667)$ con una $\mathrm{TI}$ $=52.6$, los estados que reportaron más casos fueron Veracruz, Tabasco, Tamaulipas, Nuevo León y Baja California Sur. En el sexo femenino la FD se presentó en mayor proporción con $56 \%$ y la FHD con $50 \%$. En estos años, aunque había cocirculación de los cuatro serotipos fueron predominantes DENV-1 y DENV-2.

Pese a que de 2014 a 2018 hubo una reducción importante en el número de casos de dengue, para la SE 35 de 2019 (actualización 02 de septiembre) se evidenció el marcado aumento en el número de casos, no sólo en México, sino también para muchos países de América Latina. En nuestro país hasta esta semana se tenía el reporte de un total de 11,593 casos confirmados, de los cuales 7,972 correspondieron a DNG, 2,823 a DCSA y 798 a DG con una TI hasta el momento de 9.15. Los serotipos con circulación predominante fueron DENV-1 y DENV-2; se produjeron 28 defunciones y una tasa de letalidad de 0.77 . Los estados más afectados fueron: Veracruz (3,234 casos, $\mathrm{TI}=39.08)$, Quintana Roo (592 casos, $\mathrm{Tl}=33.75)$, Chiapas $(1,792$ casos, $\mathrm{TI}=$ 32.54), Jalisco ( 1,777 casos, $\mathrm{TI}=21.46)$ y Morelos (414 casos, $\mathrm{TI}=20.60)$. El $56 \%$ de los casos de DNG, $54 \%$ de DCSA y DG se presentaron en el sexo femenino.

De manera reciente, en el año 2019, se registraron un total de 41,505 casos confirmados, de los cuales 27,884 fueron por DNG, 10,264 por DCSA y 3,357 por DG. Al igual que en la SE 35, fecha en la cual se reafirmó el incremento de casos a nivel regional, al cerrar el año los serotipos con circulación predominante fueron: DENV-1 y DENV-2. Hasta la SE $52,69 \%$ de los casos confirmados correspondieron a Jalisco $(11,727$ casos, $\mathrm{TI}=141.60)$, Veracruz $(10,902$ casos, $\mathrm{TI}=131.74)$, Chiapas $(2,241$ casos, $\mathrm{TI}=40.69)$, Quintana Roo (1,950 casos, $\mathrm{TI}=111.17$ ) y Oaxaca $(1,678$ casos, $\mathrm{TI}=40.86)$. Referente a la SE 35, en la cual Morelos (414 casos, $\mathrm{TI}=20.60$ ) 
Rev Latin Infect Pediatr 2020; 33 (2): 78-83

pertenecía a los cinco estados más afectados, al finalizar el año ocupó el octavo sitio dentro de los estados con mayor número de casos con 1,420 casos confirmados y una TI de 70.67. A la última semana del año, el sexo femenino continuó presentando el mayor porcentaje de casos tanto de DNG con 57\% como para DCSA con $55 \%$; finalmente los grupos de edad más afectados fueron de 10-14 y de 15-19 años.

\section{DISCUSIÓN}

Aunque el presente trabajo proporciona una visión general del comportamiento epidemiológico de los casos confirmados de la enfermedad del dengue para México durante el periodo 2000-2019, como en muchos sistemas de vigilancia del mundo, los casos que se reportan son únicamente los que se notifican a los servicios de salud, por lo que se desconoce con certeza los casos y las tasas de incidencia verdaderamente atribuibles al virus del dengue.

La incidencia de dengue ha aumentado de manera constante en México, de cerca de 2.4 casos por cada 100,000 habitantes en el año 2000 a 52.6 casos en 2013, y aunque a partir de este año las tasas de incidencia disminuyeron en un $50-70 \%$ aproximadamente, en 2019 a partir de la SE 20 comenzó a presentarse un incremento en el número de casos confirmados y al cerrar el año 2019 (SE 01 a la 52) se registraron más de 41 mil casos, que en comparación con los 12,706 casos al cierre del año
2018 representó un aumento del $226 \%$ de casos. ${ }^{16,18}$ De la misma manera, la TI calculada para 2018 fue de 10.13, menor con respecto a 32.79 en 2019; en cuanto al número de defunciones fueron reportadas 75 versus 191, respectivamente.

Estos datos demuestran que el comportamiento del dengue a lo largo de los años es dinámico y que a pesar de los constantes esfuerzos por controlar el vector y mitigar la transmisión del virus existen ciertos patrones de los brotes que coinciden con los que se presentan a nivel regional e inclusive mundial, ${ }^{19,20}$ es decir, la distribución geográfica puede ser el resultado de características climáticas como la temperatura y la humedad con modificaciones en el ámbito ecológico, que han contribuido a la expansión de los vectores Aedes aegypti y A. albopictus. Por otro lado, factores demográficos como el aumento en la densidad de la población, migración humana y turismo favorecen también a la transmisión de casos de dengue..$^{21}$ Tal es el caso de la situación actual, pues no sólo en México se ha presentado este incremento de casos en 2019, sino también de forma global después del descenso observado en el número de casos reportados de dengue en 2017 y $2018 .{ }^{4}$

En la Región del Pacífico Occidental este aumento se ha observado en Australia, Camboya, China, República Democrática Popular Lao, Malasia, Filipinas, Singapur y Vietnam, mientras que se han notificado casos por DEN-2 en Nueva Caledonia, y por DEN-1 en la Polinesia francesa. En la Región de África también se han notificado brotes en el Congo,

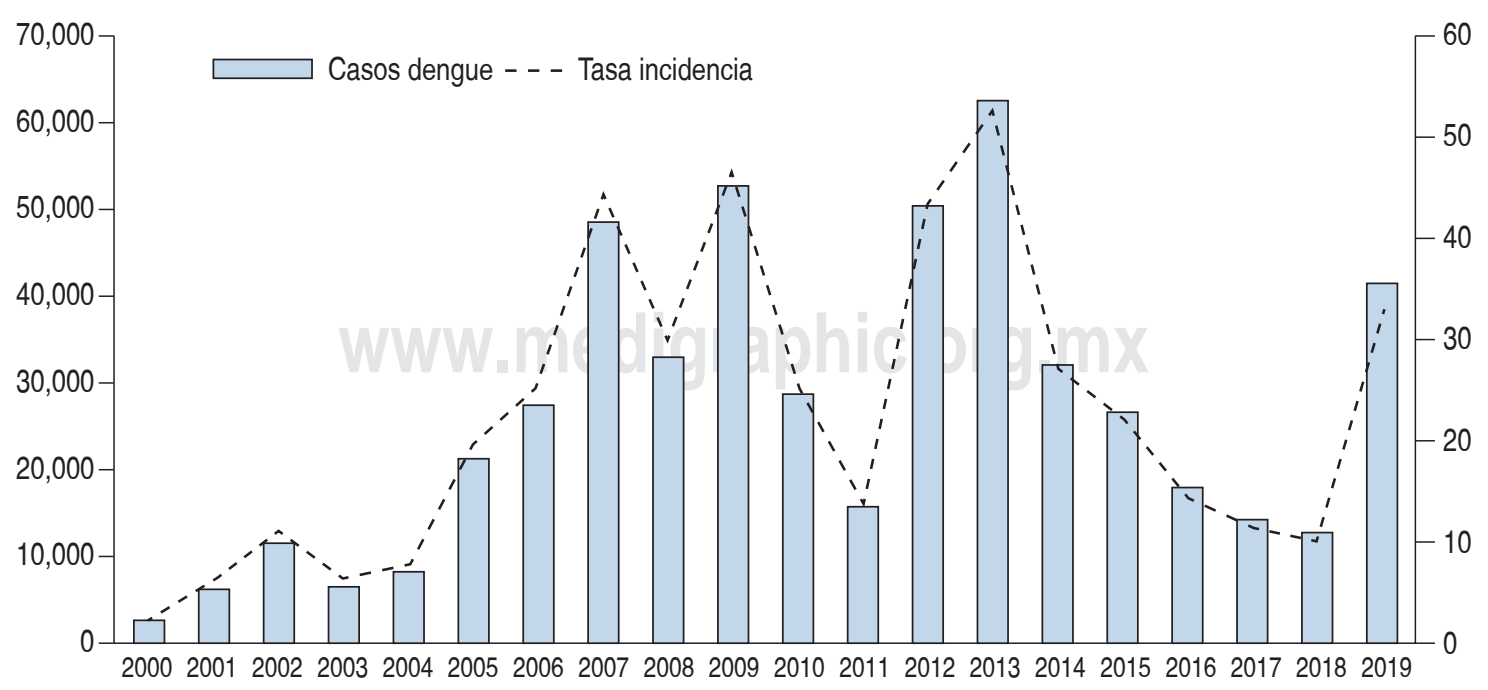

Figura 2: Casos confirmados de dengue y tasa de incidencia anual en México de 2000 a 2019. ${ }^{15-17}$ 
Rev Latin Infect Pediatr 2020; 33 (2): 78-83

Côte d'Ivoire y Tanzania. ${ }^{22}$ De la misma manera, en algunos países de la Región de las Américas se ha observado un aumento del número de casos. Según la actualización epidemiológica (11 de noviembre) más reciente publicada por la Organización Panamericana de la Salud (OPS), entre la SE 01 y la SE 42 de 2019 en esta región se alcanzó el mayor número de casos registrados en la historia con cerca de 2,733,635 casos (incidencia de 280 casos por 100,000 habitantes), incluyendo 1,206 defunciones. De estos casos, 1,217,196 (44.5\%) fueron confirmados por criterio de laboratorio y $22,127(0.8 \%)$ fueron clasificados como DG, mientras que la tasa de letalidad fue de $0.04 \%$. El total de casos reportados comprendidos en este periodo fue superior al total registrado durante los años 2016, 2017 y 2018. ${ }^{23}$

En esta actualización también se reportó que los cuatro serotipos estuvieron presentes en las Américas con la circulación simultánea de todos ellos en Brasil, Guatemala y México, lo cual aumenta la ocurrencia de casos graves de la enfermedad, mientras que en Colombia, Guadalupe, Panamá, Martinica y Venezuela circularon los serotipos DENV1, DENV-2 y DENV-3; y en Paraguay y Perú DENV-1, DENV-2 y DENV4. ${ }^{23}$

Los países de las Américas con las tasas de incidencia más altas fueron: Brasil con 2,069,505 casos reportados, México con 181,625 casos, Nicaragua con 142,740 casos, Colombia con 101,129 casos y finalmente Honduras con 91,681. 4,22 Sin embargo, los países con las TI más altas fueron en su mayoría Centroamericanos: Nicaragua $(2,271.12$ casos por 100,000 habitantes), Belice $(1,021.20$ casos por 100,000 habitantes), Honduras (995.51 casos por 100,000 habitantes) y El Salvador (375 casos por 100,000 habitantes). El quinto país con mayor tasa de incidencia en las Américas fue Brasil con 711.2 casos por 100,000 habitantes. ${ }^{23}$

Estos datos confirman que, a nivel mundial, en la Región de las Américas y en México, la enfermedad de dengue sigue siendo un problema de salud pública y aunque su comportamiento epidemiológico sigue un patrón cíclico de brotes influenciado por múltiples factores, es imperante tener mejores estrategias de vigilancia, diagnóstico y control integrado de vectores que mitiguen su impacto.

\section{REFERENCIAS}

1. Murray NE, Quam MB, Wilder-Smith A. Epidemiology of dengue: past, present and future prospects. Clin Epidemiol. 2013; 5: 299-309. doi: 10.2147/CLEP.S34440.
2. Laserna A, Barahona-Correa J, Baquero L, CastañedaCardona C, Rosselli D. Economic impact of Dengue fever in Latin America and the Caribbean: a systematic review. Rev Panam Salud Publica. 2018; 42: e111. https://doi. org/10.26633/RPSP.2018.111.

3. Wilder-Smith A, Ooi EE, Horstick O, Wills B. Dengue. Lancet. 2019; 393 (10169): 350-363.

4. Organización Panamericana de la Salud (OPS). Plataforma de Información en Salud de las Américas. Dengue (PLISA 2019). [Acceso el 23 de septiembre 2019] Disponible en: http://www.paho.org/data/index.php/es/temas/indicadoresDengue.html.

5. Beltrán-Silva SL, Chacón-Hernández SS, Moreno-Palacios E, Pereyra-Molina JA. Clinical and differential diagnosis: dengue, Chikungunya and Zika. Revista Médica Del Hospital General de México. 2018; 81 (3): 146-153. doi: 10.1016/j. hgmx.2016.09.011.

6. Torres-Galicia I, Cortés-Poza D, Becker I. Dengue en México: análisis de dos décadas. Gaceta Médica de México. 2014; 150: 122-127.

7. Arredondo-García JL, Méndez-Herrera A, Medina-Cortina $\mathrm{H}$. Arbovirus en Latinoamérica. Acta Pediátrica de México. 2016; 37 (2): 111-131.

8. Organización Mundial de la Salud (OMS). Dengue: Información general. [Acceso el 03 de octubre 2019] Disponible en: https://www.paho.org/hq/index. php?option=com_content\&view $=$ article\&id $=4493: 2010$ informacion-general-Dengue\&ltemid=40232\&lang=es.

9. Organización Mundial de la Salud (OMS). Dengue y Dengue grave 2019. [Acceso el 03 de octubre 2019] Disponible en: https://www.who.int/es/news-room/fact-sheets/detail/ Dengue-and-severe-Dengue.

10. Organización Panamericana de la Salud/Organización Mundial de la Salud. Guías de atención para enfermos de Dengue en la región de las Américas, 2010. [Acceso el 03 de octubre 2019] Disponible en: http://www.paho.org/hq/ index.php?option=com_docman\&task=doc_view\&gid=1123 9\&ltemid=270\&lang=en.

11. SINAVE/DGE/Secretaria de Salud. ¿Sabes qué es el Sistema Nacional de Vigilancia Epidemiológica? Elemento fundamental para la salud pública. [Acceso el 30 septiembre 2019] Disponible en: https://www.gob.mx/salud/ articulos/sabes-que-es-el-sistema-nacional-de-vigilanciaepidemiologica.

12. Short EE, Caminade C, Thomas BN. Climate change contribution to the emergence or reemergence of parasitic diseases. Infect Dis. 2017; 10: 1178633617732296. doi: $10.1177 / 1178633617732296$.

13. Kesetyaningsih TW, Andarini S, Sudarto, Pramoedyo $\mathrm{H}$. Determination of environmental factors affecting Dengue incidence in sleman district, yogyakarta, indonesia. Afr J Infect Dis. 2018; 12 (1 Suppl): 13-25. doi: 10.2101/ Ajid.12v1S.3.

14. Teurlai M, Menkès CE, Cavarero V, Degallier N, Descloux E, Grangeon JP et al. Socio-economic and climate factors associated with dengue fever spatial heterogeneity: a worked example in New Caledonia. PLoS Negl Trop Dis. 2015; 9 (12): e0004211. doi: 10.1371/journal.pntd.0004211.

15. SINAVE/DGE/Secretaria de Salud. Histórico Boletín Epidemiológico 2000-2018. [Acceso 17 septiembre 2019] Disponible en: https://www.gob.mx/salud/acciones-yprogramas/historico-boletin-epidemiologico.

16. SINAVE/DGE/Secretaria de Salud. Boletín Epidemiológico 2019. [Acceso 17 septiembre 2019] Disponible en: https:// 
Rev Latin Infect Pediatr 2020; 33 (2): 78-83

www.gob.mx/salud/documentos/_-boletinepidemiologicosistema-nacional-de-vigilancia-epidemiologica-sistemaunico-de-informacion-186989.

17. SINAVE/DGE/Secretaria de Salud. Panorama Epidemiológico de Dengue 2019. [Acceso 17 septiembre 2019] Disponible en: https://www.gob.mx/cms/uploads/ attachment/file/490854/Pano_Dengue_35_2019.pdf.

18. SINAVE/DGE/Secretaria de Salud. Panorama Epidemiológico de Dengue 2018. [Acceso 17 septiembre 2019] Disponible en: https://www.gob.mx/salud/ acciones-y-programas/panorama-epidemiologico-deDengue-2018.

19. Abd Majid N, Muhamad Nazi N, Mohamed AF. Distribution and spatial pattern analysis on dengue cases in seremban district, negeri sembilan, Malaysia. Sustainability. 2019; 11 (13): 3572. doi: 10.3390/su11133572.

20. Bennett SN, Drummond AJ, Kapan DD, Suchard MA, Muñoz-Jordán JL, Pybus OG et al. Epidemic dynamics revealed in Dengue evolution. Mol Biol Evol. 2010; 27 (4): 811-818. doi: 10.1093/molbev/msp285.
21. Dantés HG, Farfán-Ale JA, Sarti E. Epidemiological trends of dengue disease in Mexico (2000-2011): a systematic literature search and analysis. PLoS Negl Trop Dis. 2014; 8 (11): e3158. doi: 10.1371/journal.pntd.0003158.

22. Organización Panamericana de la Salud/Organización Mundial de la Salud. Actualización Epidemiológica: Dengue. 13 de septiembre de 2019, Washington, D.C. OPS/OMS. 2019.

23. Organización Panamericana de la Salud/Organización Mundial de la Salud. Actualización Epidemiológica: Dengue. 11 de noviembre de 2019, Washington, D.C. OPS/OMS. 2019.

Correspondencia:

José Luis Arredondo García

Jefe de la Unidad de Investigación Clínica,

Instituto Nacional de Pediatría

Insurgentes Sur Núm. 3700-C,

Col. Insurgentes Cuicuilco, 04530,

Alcaldía Coyoacán, Ciudad de México, México.

E-mail: gabriela.aguilar.le@gmail.com 